VOL. $61(2000)$ [523-524]

\title{
Metacyclic groups
}

\author{
Charles E. Hempel
}

A group $G$ is metacyclic if it contains a cyclic, normal subgroup $N$ such that $G / N$ is also cyclic. It was already known to Hölder that finite metacyclic groups can be presented on two generators and three defining relations.

Certain classes of metacyclic groups have been given much attention. Zassenhaus discussed metacyclic groups with cyclic commutator quotient, as did M. Hall. Beyl, King, Liedahl, Newman and Xu, Rédei and Lindenberg all classified metacyclic $p$ groups of odd order.

Significant progress was made in 1994 when Hyo-Seob Sim proved in [2] that every finite metacyclic group decomposes naturally as a semidirect product of two Hall subgroups. The normal semidirect factor is chosen as the smallest normal Hall subgroup with nilpotent quotient; it was shown that it is always a split metacyclic group of odd order. The other semidirect factor is nilpotent. By breaking the problem into two simpler problems, Sim proceeded to classify metacycic groups of odd order. In Chapter 2 of my thesis some of his key contributions are recalled.

The difficulty in generalising from groups of odd order to arbitrary groups lies in the metacyclic 2-groups. First they needed to be classified up to isomorphism. In Chapter 3, a variant of a classification taken from unpublished work of Newman and Xu [1] done in 1988 is presented. Although metacyclic 2-groups have been classified, little of their automorphism groups was known. In Chapter 4 a type of automorphism group of 2 -groups is calculated with the aid of the classification of Chapter 3. Finally, finite metacyclic groups are classified in Theorem 5.3. Those groups which are split are then identified. A computer program written in GAP to determine the canonical presentation afforded by Theorem 5.3 of any finite metacyclic group is listed in Chapter 6 . Infinite metacyclic groups, the structure of which is by comparison much easier to understand, are classified in terms of presentations in Chapter 7.

Received 10th January, 2000

Thesis submitted to the Australian National Unviersity, May 1998. Degree approved, December 1998. Supervisor: Dr L.G. Kovács.

Copyright Clearance Centre, Inc. Serial-fee code: 0004-9727/00 \$A2.00+0.00. 


\section{REFERENCES}

[1] M.F. Newman and M. Xu, 'Metacyclic groups of prime-power order', Adv. Math. 17 (1988), 106-107.

[2] H-S. Sim, 'Metacyclic groups of odd order', Proc. London Math. Soc. (3) 69 (1994), 47-71.

8/39 Roslyn Gardens

Elizabeth Bay NSW 2011

Australia 